\title{
Video Article \\ Combining Magnetic Sorting of Mother Cells and Fluctuation Tests to Analyze Genome Instability During Mitotic Cell Aging in Saccharomyces cerevisiae
}

\author{
Melissa N. Patterson ${ }^{1}$, Patrick H. Maxwell ${ }^{1}$ \\ ${ }^{1}$ Department of Biological Sciences, Rensselaer Polytechnic Institute \\ Correspondence to: Patrick H. Maxwell at maxwep2@rpi.edu
}

URL: https://www.jove.com/video/51850

DOI: doi: $10.3791 / 51850$

Keywords: Microbiology, Issue 92, Aging, mutations, genome instability, Saccharomyces cerevisiae, fluctuation test, magnetic sorting, mother cell, replicative aging

Date Published: 10/16/2014

Citation: Patterson, M.N., Maxwell, P.H. Combining Magnetic Sorting of Mother Cells and Fluctuation Tests to Analyze Genome Instability During Mitotic Cell Aging in Saccharomyces cerevisiae. J. Vis. Exp. (92), e51850, doi:10.3791/51850 (2014).

\section{Abstract}

Saccharomyces cerevisiae has been an excellent model system for examining mechanisms and consequences of genome instability. Information gained from this yeast model is relevant to many organisms, including humans, since DNA repair and DNA damage response factors are well conserved across diverse species. However, S. cerevisiae has not yet been used to fully address whether the rate of accumulating mutations changes with increasing replicative (mitotic) age due to technical constraints. For instance, measurements of yeast replicative lifespan through micromanipulation involve very small populations of cells, which prohibit detection of rare mutations. Genetic methods to enrich for mother cells in populations by inducing death of daughter cells have been developed, but population sizes are still limited by the frequency with which random mutations that compromise the selection systems occur. The current protocol takes advantage of magnetic sorting of surface-labeled yeast mother cells to obtain large enough populations of aging mother cells to quantify rare mutations through phenotypic selections. Mutation rates, measured through fluctuation tests, and mutation frequencies are first established for young cells and used to predict the frequency of mutations in mother cells of various replicative ages. Mutation frequencies are then determined for sorted mother cells, and the age of the mother cells is determined using flow cytometry by staining with a fluorescent reagent that detects bud scars formed on their cell surfaces during cell division. Comparison of predicted mutation frequencies based on the number of cell divisions to the frequencies experimentally observed for mother cells of a given replicative age can then identify whether there are age-related changes in the rate of accumulating mutations. Variations of this basic protocol provide the means to investigate the influence of alterations in specific gene functions or specific environmental conditions on mutation accumulation to address mechanisms underlying genome instability during replicative aging.

\section{Video Link}

The video component of this article can be found at https://www.jove.com/video/51850/

\section{Introduction}

Microorganisms, such as the yeast Saccharomyces cerevisiae, are excellent models for investigating mutation rates, but these models have not been fully utilized to investigate the accumulation of mutations during mitotic aging of cells. Mutation accumulation in the nuclear genome has been hypothesized to contribute to aging by resulting in progressive loss of (or variation in) gene functions ${ }^{1}$. The ability to use a microbial system to study the mechanisms and consequences of mutation accumulation during aging could greatly accelerate the identification of genetic and environmental factors influencing this process, because of facile genetic systems and the ease of quantifying rare phenotypic changes in microorganisms. DNA repair factors and DNA damage response proteins are well conserved across diverse species ${ }^{2}$, and fundamental similarities in organismal aging have been identified for organisms as diverse as S. cerevisiae and mammals ${ }^{3}$, making it likely that results obtained from studies in yeast will be relevant to aging in many organisms.

Studies of aging in S. cerevisiae make use of two aging models that measure chronological aging or replicative aging of cells. Chronological aging is characterized by the progressive loss of viability in yeast cell populations that are in a non-dividing state (stationary phase) due to nutrient depletion ${ }^{3}$. The chronological aging model has been used to demonstrate that mutations accumulate with increasing age ${ }^{4}$, since large cell populations are easily obtained in this model to perform phenotypic selections for mutant cells. In this case, mutation accumulation appears to be influenced by growth-signaling pathways and oxidative stress ${ }^{5}$, and each of these factors is relevant to understanding aging in multicellular eukaryotes ${ }^{3}$. The replicative aging model exploits the asymmetric division between S. cerevisiae mother and daughter cells for investigating aging that occurs with successive cell generations ${ }^{3}$. Yeast replicative aging has often been measured through micromanipulation of small populations of mother and daughter cells, or more recently, through video microscopy of small populations of yeast cells in microfluidic devices $^{6,7}$. Limited population sizes make these approaches poorly suited for investigating mutation accumulation during mitotic aging unless whole-genome information is obtained from single cells or very high frequency genetic changes are measured ${ }^{8}$. Whole-genome sequencing of single cells provides substantial data sets for investigating mutation accumulation, but phenotypic selection systems have the important advantage of providing a means of measuring mutation rates to study mechanisms underlying mutation accumulation. Studies to examine mutation frequencies and rates through phenotypic selections in haploid yeast strains during replicative aging have not yet been reported. 
Mutation rates are commonly measured using fluctuation tests ${ }^{9}$. Mutation frequency values reflect the total number of cells harboring a mutation in a gene sequence in a population. This includes cells in which independent mutations occur and any progeny of those cells that simply inherit pre-existing mutations. In contrast, mutation rates measured through fluctuation tests represent the number of independent mutations that newly arise with each cell generation. These tests typically involve inoculating many replicate cultures at low cell densities to reduce the likelihood of adding cells with pre-existing mutations in a target sequence, growing the cultures to near saturation, and identifying mutant cells by growth on selective medium. The numbers of mutant cells identified in the replicate populations are then used in one of several mathematical models to estimate the number of independent mutations that arose during growth ${ }^{9}$. Comparing the number of independent mutations to the average population size provides a measure of the mutation rate. In $S$. cerevisiae, mutation frequencies and rates are frequently measured using the URA3 and CAN1 genes, since loss-of-function mutations in these genes produce selectable phenotypes. Loss of URA3 function renders cells resistant to 5 -fluoroorotic acid (5-FOA), since the protein encoded by URA3 converts 5-FOA into a toxic molecule ${ }^{10}$. Loss of function of CAN1 allows cells to become resistant to canavanine, a toxic arginine analog, since the protein encoded by CAN1 transports arginine and canavanine into cells ${ }^{11}$.

Both genetic and physical sorting strategies have been successfully employed to obtain larger populations of S. cerevisiae mother cells to facilitate investigations of replicative aging. Genetic strategies include clever approaches that select against daughter cell survival in a population. The mother enrichment program (MEP) involves beta-estradiol induction of Cre recombinase expression only in daughter cells, leading to daughter-specific disruption of two essential genes that were engineered to contain loxP sites ${ }^{12}$. MEP strains can be grown normally in the absence of the inducer, but only the mother cells will continue to divide following induction, while daughter cells will arrest at M-phase typically during their first progression through the cell cycle ${ }^{12}$. While this system has not been used to broadly study mutation accumulation during aging, it has been used to study loss of heterozygosity, a relatively frequent form of genome instability in diploid yeast strains, as well as biochemical changes in aging mother cells ${ }^{13,14}$. Similarly, the daughter-arrester system involves daughter-specific expression of the $S$. cerevisiae URA3 gene ${ }^{15}$. Daughter-arrester strains grow normally until 5-FOA is added to the medium, at which point daughter cells expressing URA3 will die, while mother cells continue to grow ${ }^{15}$. These are useful systems to enrich for mother cells, but they depend on maintenance of the gene functions that constitute the selection system. Random mutations in one or more components of the selection system could result in daughter cells that escape the selection and proliferate exponentially. During development of the MEP strains, appropriate population sizes were determined to avoid the appearance of mutant daughter cells that could escape the selection ${ }^{12}$. However, this limit in population size compromises the detection of rare forms of genome instability during replicative aging. This restriction on population size could be partly overcome by using diploid yeast strains with two copies of each gene contributing to the selection system, since most mutations would likely be recessive ${ }^{12}$. However, use of diploid strains constrains the types of mutational events that can be easily detected compared to those that can be easily detected in haploid yeast cells.

Physical sorting strategies include isolation of mother cells with a labeled cell surface from unlabeled daughter cells to enrich for large populations of mother cells. The observation that cell surface proteins (cell wall proteins) of $S$. cerevisiae daughter cells are newly synthesized during budding has been exploited to label mother cells without labeling the daughter cells that they produce ${ }^{16}$. For example, an initial population of yeast cells labeled on their surface with biotin can be grown and then isolated from their daughter cells using anti-biotin microbeads and magnetic sorting ${ }^{16}$ because the daughter cells generated by the initial population will not contain biotin on their surface. Serial growth and sorting allows progressively older populations of mother cells to be obtained and analyzed. This procedure has been used successfully to examine changes in cell physiology and gene expression during replicative aging of yeast ${ }^{13,14,17,18}$. The current method adapts this biotin labeling and magnetic sorting technique to enrich for mother cells with the purpose of measuring the accumulation of potentially rare mutations or other forms of genome instability assayed through phenotypic selections. Serial growth and physical sorting allow analysis of mutation accumulation in progressively older mother cells, as well as in their daughter cell populations. Determination of mutation rates per generation allows a predicted mutation frequency to be calculated for mother cells that have undergone a particular number of cell divisions. Since predicted frequency values are based on the expected increase due to additional rounds of cell division, substantial deviations in the observed mutation frequencies compared to the predicted values provide evidence for age-specific changes in the rate of accumulating mutations. Using this protocol, fluorescent staining of bud scars that correspond to sites of cell divisions on mother cells coupled with analysis by flow cytometry can be used to quickly determine the age distribution of sorted and unsorted populations. Additional fluorescent reagents, such as those used for detecting reactive oxygen species, can also be utilized during this protocol. Overall, this protocol enables efficient analysis of age-dependent changes in genome instability with the potential for correlation to age-related cell physiological changes in a model organism well suited to studying the genetic and environmental factors that influence aging-related genome instability.

\section{Preparation of Media and Solutions}

1. Grow cells using yeast extract-peptone-glucose (YPD) rich medium for the standard protocol. Prepare a $2 \%$ bacto-peptone, $1 \%$ yeast extract, $2 \%$ glucose solution for YPD medium and add $2 \%$ bacto-agar for solid medium ${ }^{19}$. Autoclave to sterilize. Use an alternative medium, if needed, to test a particular growth condition or mutant yeast strain.

2. Make solid synthetic complete medium lacking arginine with $60 \mathrm{mg} / \mathrm{L}$ canavanine (SC-arg + canavanine) to select for canavanine resistant mutants. Prepare a solution that is $0.67 \%$ bacto-yeast nitrogen base without amino acids, $2 \%$ glucose, $0.2 \%$ complete supplement mixture without arginine (dropout mix), and $2 \%$ bacto-agar ${ }^{19}$. Autoclave and cool prior to adding canavanine from a $20 \mathrm{mg} / \mathrm{ml} \mathrm{stock}$ solution (filtersterilized, stored at $\left.4^{\circ} \mathrm{C}\right)^{19}$.

1. Make solid synthetic complete medium containing 5-fluoroorotic acid (5-FOA). Prepare $500 \mathrm{ml}$ of a solution that is $1.34 \%$ bacto-yeast nitrogen base without amino acids, $4 \%$ glucose, $0.4 \%$ complete supplement mixture, and $0.2 \% 5$-FOA and filter-sterilize ${ }^{19}$. Autoclave $500 \mathrm{ml}$ of a $4 \%$ bacto-agar solution, cool briefly, and then mix with the $500 \mathrm{ml}$ filter-sterilized nutrient solution. Use appropriate alternative media for other mutation assays.

3. For biotin labeling, make $500 \mathrm{ml} 1 \mathrm{x}$ phosphate buffered saline (PBS) solution containing $137 \mathrm{mM}$ sodium chloride, $2.7 \mathrm{mM}$ potassium chloride, $10.14 \mathrm{mM}$ sodium phosphate dibasic, and $1.77 \mathrm{mM}$ potassium phosphate dibasic, $\mathrm{pH} 8$. Store at $4{ }^{\circ} \mathrm{C}$ and keep on ice during use.

4. Make a fresh $5 \mathrm{mM}$ biotin stock in sterile, ultrapure water. 
5. To quench biotin labeling, make $500 \mathrm{ml}$ of $1 \times \mathrm{PBS}, 100 \mathrm{mM}$ glycine. Store at $4{ }^{\circ} \mathrm{C}$ and keep on ice during use.

6. Depending on the number of cells used and duration of the experiment, make 1-2 $L$ of bead-labeling buffer containing $1 \times$ PBS, 2 mM EDTA, $0.5 \%$ bovine serum albumin. Filter sterilize and de-gas buffer. Store at $4{ }^{\circ} \mathrm{C}$ and keep on ice during use.

7. Prepare a $0.4 \%$ trypan blue solution in $1 \times$ PBS, filter sterilize, and store at RT for use in cell viability measurements.

8. Aliquot small volumes $(\sim 100 \mu \mathrm{l})$ of a wheat germ agglutinin (WGA) fluorescent conjugate to store at $-20^{\circ} \mathrm{C}$ in foil wrapped (or opaque) microcentrifuge tubes for use in determining cell age.

\section{Determination of Mutation Rate and Frequency}

1. Establish a baseline mutation rate per cell generation using fluctuation tests for cells grown under standard culture conditions. Grow seven to eleven replicate $1 \mathrm{ml}$ cultures from an initial density of $1,000-5,000 \mathrm{cells} / \mathrm{ml}$ to early stationary phase $\left(\sim 10^{8} \mathrm{cell} / \mathrm{ml}\right)$.

1. For each replicate culture, dilute cells $1: 2,000$ and spread 1-4 $\mu$ l onto non-selective medium (YPD) to determine colony-forming-units/ $\mathrm{ml}$. Pellet remaining cells at $\sim 2,400 \mathrm{xg}$ for $1 \mathrm{~min}$ in a microcentrifuge and resuspend in $100 \mu \mathrm{l}$ water to spread onto selective medium to identify mutants. Incubate plates for three days at $30{ }^{\circ} \mathrm{C}$ prior to counting colonies (adjust incubation time as needed based on growth rate of cells).

2. Determine the number of independent mutations $(m)$ that occurred in the trial based on the number of mutant colonies $(r)$ obtained on selective medium. Use the Lea-Coulson median estimator to find $m$ by substituting the median number of mutant colonies for $r$ in the formula below ${ }^{9}$. Then substitute values for $m$ until the left side of the equation is virtually zero.

$\frac{\widetilde{r}}{m}-\ln (m)-1.24=0$

3. To determine mutation rate, first calculate the average number of viable cells spread onto selective medium by multiplying the average colony-forming-units $/ \mathrm{ml}$ of the replicate cultures and the volume of culture in $\mathrm{ml}$ spread onto selective medium. Divide the $m$ value from step 2.1 .2 by this average number of viable cells to obtain the mutation rate per cell generation.

2. Individually calculate the number of viable cells spread onto selective medium for each replicate culture in a trial as described in step 2.1.3. Divide the number of mutant colonies $(r)$ obtained for a culture by the number of viable cells spread onto selective medium to obtain the mutation frequency. Use the average or median of the individual mutation frequencies from the replicate cultures in step 2.1 as a baseline mutation frequency.

3. Make frequency measurements before and after biotin labeling cells (steps 3.2 and 3.4) to determine if the labeling step results in any change in the frequency of mutations that will need to be considered when testing for age-specific changes in mutation accumulation.

\section{Biotin Labeling}

1. Streak S. cerevisiae from a glycerol stock at $-80^{\circ} \mathrm{C}$ onto YPD medium and incubate at $30{ }^{\circ} \mathrm{C}$ for $1-3$ days.

2. Using a sterile pipette tip, transfer cells from the streak plate to $1 \mathrm{ml}$ (or more) of water keeping in mind that $1-1.5 \mathrm{~cm}$ of a thickly grown portion of the streak will give approximately $10^{8}$ cells. Determine exact cell density using a hemocytometer.

3. Label $10^{8}$ cells per strain (or treatment condition) per collection point at which mutation frequency will be determined or a population size sufficient to obtain at least 5-10 mutant colonies per sampling on selective medium (based on mutation frequency from step 2.2). Include an additional $10^{8}$ cells for baseline measurements per strain/condition.

4. Biotin label according to standard procedure from the manufacturer, except use a $5 \mathrm{mM}$ biotin reagent stock and gently rock during incubation. Spin for $5 \mathrm{~min}$ at $4{ }^{\circ} \mathrm{C}$ at $3,000 \mathrm{xg}$ for all centrifugation steps, since spinning at temperatures higher than $4{ }^{\circ} \mathrm{C}$ may lead to increased cell loss. After the final wash, suspend cells to a concentration of $10^{8}$ labeled cells $/ \mathrm{ml}$ in water.

5. Check viability of cells using trypan blue staining. Dilute $10 \mu \mathrm{l}$ of cells with $23 \mu \mathrm{l}$ of water and $67 \mu \mathrm{l}$ of $0.4 \%$ trypan blue in $1 \times$ PBS. Incubate for at least 40 min at RT before scoring live (unstained) and dead (stained) cells using a hemocytometer.

6. Measure baseline values for genome instability, cell age, and other relevant characteristics (see sections 5,6 , and 7 ).

7. Transfer biotin-labeled cells to $20 \mathrm{ml}$ of YPD medium per $10^{8}$ cells in an Erlenmeyer flask (approximately $5 \times 10^{6} \mathrm{cells} / \mathrm{ml}$ ). Grow culture(s) $16-18 \mathrm{hr}$ at $20^{\circ} \mathrm{C}$ to an approximate density of $10^{8} \mathrm{cells} / \mathrm{ml}$ (four to five cell generations), but do not allow cells to reach stationary phase. Use a shorter incubation time for growth at $30^{\circ} \mathrm{C}$. Keep biotin-labeled cells at $4{ }^{\circ} \mathrm{C}$ for up to several hours prior to addition to medium, if needed to optimize timing of the growth period and collection.

\section{Bead Labeling and Magnetic Sorting}

1. After the growth phase, dilute an aliquot of culture to determine the cell density using a hemocytometer. Pellet cells by centrifugation for 5 min at $4{ }^{\circ} \mathrm{C}$ at $3,000 \times \mathrm{g}$. Pour off supernatant.

2. Wash cells by vortexing to suspend pellet in $30 \mathrm{ml}$ of bead-labeling buffer, then centrifuging and pouring off supernatant as in section 4.1 . Repeat wash.

3. Fully resuspend cells in $50 \mu$ l bead-labeling buffer per $10^{8}$ total cells by vortexing. Add $2 \mu$ magnetic beads per $10^{8}$ total cells and vortex briefly to mix. Incubate on ice for $10 \mathrm{~min}$, inverting periodically to mix (modified from an online protocol at http://www.sysbio.org/ dataresources/usermanual031113.pdf).

4. Wash cells three times with $30 \mathrm{ml}$ of bead-labeling buffer.

5. Add $0.5 \mathrm{ml}$ bead-labeling buffer per $10^{8}$ total cells and briefly vortex pellet on medium setting just until cells are resuspended. Pour through a $40 \mu \mathrm{m}$ cell strainer into a $50 \mathrm{ml}$ tube to remove any remaining cell clumps. If needed, leave cells on ice for $1-2 \mathrm{hr}$ prior to loading on the column.

6. Follow the manufacturer's recommended protocol for the magnetic columns to bind, wash, and elute the magnetic bead-labeled mother cells For increased recovery of labeled cells, use at least one column per $2 \times 10^{9}$ total cells.

1. Remove any bubbles that occur when loading columns, as they will block flow. Remove and replace the column on the separator between washes to prevent cells from becoming trapped between the beads (modified from an online protocol at http://www.sysbio.org/ dataresources/usermanual031113.pdf) 
2. Save flow through from binding and washing steps to analyze young cells produced by the mother cells, if desired. Concentrate cells as described in step 4.7.

3. Use multi-column separators for processing multiple samples at once. Elute multiple columns of the same sample into the same collection tube to reduce handling time and decrease loss of cells.

7. Concentrate eluted mother cells by centrifuging at $3,000 \mathrm{xg}$ for $5 \mathrm{~min}$ at $4{ }^{\circ} \mathrm{C}$. Aspirate all but $1 \mathrm{ml}$ buffer per $10^{8}$ original biotin-labeled cells Vortex briefly to suspend cells in remaining buffer.

8. Dilute an aliquot of cells and stain with trypan blue to determine the cell density and cell viability using a hemocytometer (see step 3.5). Calculate the total number of cells recovered.

1. If the number of cells is substantially higher than expected, pass the elution sample through another column to try to remove contaminating young cells. If the number of cells is substantially lower than expected, pass the saved flow through sample through another column to try to improve yield of mother cells.

9. Use cells for further analyses, as described in sections 5 and 6 . Alternatively, grow the cells in YPD broth again to increase their replicative age (step 3.7) and follow by bead labeling and sorting (steps 4.1-4.8.1), without the need to repeat the biotin labeling.

\section{Determination of Replicative Age}

1. Spin cells for $2 \mathrm{~min}$ at $5,000 \times \mathrm{g}$ at RT for all steps in this section. Put $25 \mu \mathrm{l}$ of recovered cells $\left(\sim 10^{8}\right.$ cells $\left./ \mathrm{ml}\right)$ into a $1.5 \mathrm{ml}$ centrifuge tube and wash twice with $1 \mathrm{ml} 1 \times$ PBS. Aspirate off supernatant.

1. Thaw an aliquot of WGA-conjugate, vortex to mix, and spin briefly to eliminate protein aggregates. Suspend cells in $180 \mu \mathrm{l} 1 \mathrm{x}$ PBS and $20 \mu \mathrm{l}$ of WGA conjugated to a fluorescent molecule to label bud scars on the surface of the cells. Cover the $1.5 \mathrm{ml}$ centrifuge tube with foil and incubate with gentle rocking at $30^{\circ} \mathrm{C}$ for $30 \mathrm{~min}$.

2. Wash three times with $1 \mathrm{ml} 1 \mathrm{X}$ PBS.

2. For fluorescent microscopy, label slides and mount per standard protocol using an anti-fade agent. Fully cure slides in the dark (up to a few days) before sealing coverslip to avoid cell movement during imaging. Count bud scars on at least 50 cells per sample to obtain a representative measure of cell age. Store slides for up to 1 month for viewing bud scars, if needed.

1. Alternatively, determine the intensity of the fluorescent signal from the WGA-conjugate by performing flow cytometry on cells suspended in $1 \mathrm{ml}$ 1x PBS after step 5.1.2. Use a $405 \mathrm{~nm}$ excitation laser and 530/30 nm emission filter with a $505 \mathrm{~nm}$ long pass filter for WGA-Alexa 488. Include an unstained control for each collection point.

3. Graph bud scars on young cells and aged cells counted by microscopy on the y-axis against the normalized geometric mean (stained geometric mean/unstained geometric mean) of the WGA-conjugate fluorescence from the same cell populations on the x-axis. Obtain the equation of a linear trend line for this relationship. For subsequent experiments, substitute the normalized geometric mean of WGA-conjugate fluorescence intensity of a cell population for $\mathrm{x}$ in the equation and solve for $\mathrm{y}$ to determine the average cell age of a sample.

\section{Mutation Frequency}

1. Spread sorted mother cells resuspended in buffer from step 4.7 onto YPD medium and selective medium as described in step 2.1.1. Use $1 \mathrm{ml}$ of resuspended mother cells for selective medium and spin cells at 5,000 $\mathrm{g}$ for $1 \mathrm{~min}$.

NOTE: Spread larger volumes of diluted cells onto YPD medium as cells increase in age to compensate for increasing numbers of non-viable and senescent cells in older populations.

1. Incubate YPD medium and selective medium plates from step 6.1 at $30^{\circ} \mathrm{C}$ for 3 and 4 days, respectively. Increase incubation time up to one week for very old cells or slowly growing strains. Calculate mutation frequency as described in step 2.2.

\section{Calculating Predicted Mutation Frequency for a Given Replicative Age}

1. Calculate the average increase in replicative age of the biotin-labeled cells over the course of the experiment by subtracting the average age of the initial cell population from the average age of the sorted mother cells for the relevant sort. Use the cell ages calculated in step 5.2 or step 5.2.2.

2. Multiply the mutation rate obtained in step 2.1 .3 by the increase in average cell age calculated in step 7.1 . Add this value to the baseline mutation frequency for the initial population calculated in step 2.2 to determine the predicted mutation frequency for cells of the relevant replicative age.

\section{Representative Results}

A flow diagram in Figure 1 shows the overall steps of the procedure, including the points at which mutation rates, frequencies, and cell age are measured. Accurately determining the frequency and rate of mutations (or other form of genome instability) in young cell populations is an important first step, since it is necessary for choosing an appropriate population size for labeling and magnetic sorting. Rate values can be established using fluctuation tests ${ }^{9}$. Example results for yeast strains in the BY4741 genetic background ${ }^{20}$ selected for mutations in the CAN1 gene that confer canavanine resistance or mutations in the URA3 gene that confer resistance to 5-FOA are shown in Figure 2. Cells were grown at $20^{\circ} \mathrm{C}$ for representative rate experiments, and at $20^{\circ} \mathrm{C}$ and $30{ }^{\circ} \mathrm{C}$ for representative frequency experiments. These temperatures were used because initial cell populations grown at $30^{\circ} \mathrm{C}$ were then grown at $20^{\circ} \mathrm{C}$ for the subsequent representative sorting experiments. Rate values from independent trials were comparable for the initial cell population and cells after biotin labeling for a strain that has the CAN1 gene at its normal location on chromosome V, approximately 32 kilobase pairs from the left arm telomere (www.yeastgenome.com) (Figure 2A, lavender columns). The mutation rate of CAN1 was substantially higher in initial populations of a second yeast strain that has a deletion of the CAN1 
gene from its normal location on chromosome $\mathrm{V}$ and an insertion of CAN1 on the right arm of chromosome VIII approximately 25 kilobase pairs from the telomere ${ }^{21}$ (Figure 2B). CAN1 gene mutation frequencies were also comparable for initial cell populations and biotin labeled cells at either growth temperature (Figure 2A, blue columns). However, mutation frequencies obtained at $20^{\circ} \mathrm{C}$ were found to be higher than mutation frequencies at $30^{\circ} \mathrm{C}$. To test whether this temperature effect was limited to the CAN1 gene, we measured mutations in URA3 by selecting for resistance to 5-FOA using the yeast strain that has CAN1 inserted on chromosome VIII. This strain also has a deletion of URA3 from its normal site on chromosome $\mathrm{V}$ and an insertion of URA3 onto the right arm of chromosome VIII approximately 40 kilobase pairs from the telomere ${ }^{21}$ Mutation frequencies were also higher at $20^{\circ} \mathrm{C}$ for URA3 at this chromosomal location (Figure 2C). Overall, this demonstrates that growth temperature can affect the frequency of mutations, but biotin labeling does not appear to affect mutation frequency. Therefore, mutation rates and frequencies for initial populations need to be determined at the same growth temperature that will be used for rounds of growth and sorting. It is advisable to verify that biotin labeling does not influence genome instability prior to using the protocol to investigate other types of mutations or genome rearrangements.

As expected, the mutation rate values shown in Figure 2A are several-fold lower than the corresponding frequency measurements. This difference occurs because mutation rate measures the appearance of cells with new mutations with each round of cell division, while mutation frequency measures the cumulative number of mutant cells in the population at the end of the growth period. The rates and $95 \%$ confidence intervals for these trials show that reproducible results can be obtained by using seven replicate cultures per trial, which is the minimum number of replicates suggested for this protocol.

Once the initial frequency and rate values are determined, a population size should be chosen that ensures that adequate cells are present after multiple rounds of sorting to reliably determine mutation frequencies. Labeling a population of $10^{8}$ cells per time point that is to be analyzed during aging is appropriate when frequencies and rates are similar to those shown in Figure 2A. This decision also depends on how efficiently labeled mother cells are recovered after each round of sorting. The efficiency of recovering labeled mother cells can be quickly and simply evaluated by using a hemocytometer to determine the number of cells eluted from the columns for each sort and to examine cell morphology. Two main points are illustrated by the example recovery efficiency data (Figure 3A): the recovered numbers of cells may appear higher than expected after the first sort, and a small progressive loss of cells is expected with continued sorting. The higher than expected number of cells in some experiments after the first sort could result from the labeling of buds on cells in the initial population. A yeast cell with a bud would typically be scored as a single cell when determining the number of cells to label with biotin. Labeling of buds present in the initial population, though, would allow the cells that develop from those buds to also be retained on columns during sorting. The influence of this occurrence on the determination of recovery efficiency depends on the fraction of budded cells in the initial cell population. A second potential reason for the higher cell number after sort one is that there tends to be more large budded cells at this time point that may be completing cell division during the sorting. As a result, large buds still attached to their mother cells may be sorted but then appear as distinct cells when the eluted cells are examined. While some loss of cells is observed with each round of growth and sorting, the protocol can result in reliable retention of $80-90 \%$ of cells after each round of sorting. It is worth the effort to practice the procedure to ensure that $80 \%$ or more of the labeled cells are being isolated to avoid the need to label an unnecessarily large initial cell population. Treatment of cells with a mild stress after the first sort (1 mM hydrogen peroxide in YPD medium for $30 \mathrm{~min}$ ) did not prevent efficient recovery of cells with continued rounds of growth and sorting, though there was some variability in recovery for the first sort after the stress (Figure $3 \mathrm{~A}$ ).

Inspection of cell morphology and viability are also useful both for confirming successful isolation of mother cells and for adjusting dilutions/ volumes used when spreading cells on non-selective medium to determine the density of colony forming units. Mother cells become increasingly larger and more irregularly shaped as they age, compared to daughter cells (Figure 3B). The mother cell samples should therefore primarily consist of large, irregularly shaped cells as the experiment progresses through each round of sorting. The presence of many small oval cells of regular shape could indicate that mother cells are not being adequately separated from daughter cells. Viability of the mother cells should also progressively decline with each round of growing and sorting, though there may not be much change during the first five to ten cell generations. The number of cells capable of forming colonies can decrease much more dramatically than the number of cells that are determined to be viable by some form of direct staining for cell integrity, due to formation of senescent cells. When viability by a direct staining method first begins to decline (approximately $80 \%$ or less), it may be necessary to adjust dilutions and volumes used to measure colony forming unit densities in anticipation of a more dramatic decrease in the ability of the viable cells to form colonies (a two to several fold decrease).

The replicative ages of the sorted populations and control populations need to be determined before mutation frequency data from mother cells can be analyzed for age-specific changes in the rate of accumulating mutations. This can be accomplished through manual counting of bud scars on mother cells (Figure 4) or through flow cytometry to quantify the signal for the bud scar detection reagent (Figure 5). Bud scars can be labeled with relatively low background signal using WGA-fluorescent conjugates (Figure 4C). Figure 4A shows that most cells from the flow through samples of the sorting procedure have zero or one bud scar. The cells eluted from columns are mostly aged mother cells (Figures 4B and 5). Typically, $>90 \%$ of eluted cells are mother cells, which can be seen more easily from the flow cytometry result in Figure 5. Cells with relatively few bud scars $(<6)$ obtained after more than two rounds of sorting could represent contaminating cells that were not biotin labeled that underwent a few rounds of cell division during the relevant round of growth, as opposed to mother cells that are dividing very slowly. The variation in cell age may increase with subsequent rounds of sorting if not all cells in the population are growing uniformly.

A larger cell population can be used more quickly to evaluate cell age if a linear relationship is established between WGA-fluorescence signal intensities and the number of bud scars per cell. For this analysis, normalization of all WGA-fluorescence signal intensities is accomplished by dividing the signal of stained cells by the signal obtained for the appropriate unstained cell population (daughter or mother) to account for increased background fluorescence in the older cells. Figures $\mathbf{4}$ and $\mathbf{5}$ show analysis of the same representative cell populations. Manual counting established average replicative ages of 0.95 and 11.4 for the daughter cell (Figure 4A) and mother cell populations (Figure 4B), respectively. Normalized WGA-signals for these two populations and the three additional populations (average ages of 3.0,6.9, and 14.4) were plotted against the average number of bud scars for each population (Figure 5B). This linear relationship can then be used with the same strain and reagents in future experiments to determine average cell age from the normalized WGA-signal obtained through flow cytometry, allowing quick and accurate determination of cell age. Control populations should still be included to verify similar staining efficiencies between trials.

The influence of age on the accumulation of mutations can be addressed once the prior steps of obtaining mutation rate values, efficiently sorting cells, and determining cell ages are accomplished. The number of time points at which the frequency can be determined depends on the size of the biotin-labeled cell population and the number of cells that need to be spread on selective medium to obtain reliable results. If mutation rate 
changes with age, then the observed mutation frequencies for aging mother cells should differ from those expected based solely on additional rounds of cell division. Comparison of the observed mutation frequency to the predicted mutation frequency can identify age-related differences in the rate of accumulating mutations. As described in section 7 of the protocol, the predicted frequency can be obtained from the product of the baseline mutation rate for the initial cell population and the increase in replicative age of the mother cells added to the mutation frequency for the initial population. Increases in CAN1 mutation frequency have been observed with increased average cell age in a strain with CAN1 at its normal location on chromosome V (Figure 6A) and in a strain with CAN1 on the right arm of chromosome VIII (Figure 6B). Since the observed mutation frequencies for mother cells in Figure $\mathbf{6 A}$ are similar to or just below the predicted frequencies, the data do not provide any evidence for an agespecific change in mutation rate. In contrast, mutation frequencies for mother cells of the strain with CAN1 on chromosome VIII were higher than the predicted frequencies (Figure 6B). Note that the predicted frequencies in the graph do not appear to increase very much because a log scale had to be used for the $y$-axis due to the large increase in observed mutation frequency. Therefore, the results in Figure 6B do provide evidence supporting an age-specific increase in the rate of accumulating mutations. The difference in the results for the data sets in Figure 6A and 6B is likely due to the different genomic locations of CAN1. These types of observations provide a starting point for developing hypotheses to study mechanisms that affect mutation rates as cells age and to develop models to explain mutation accumulation with replicative age.

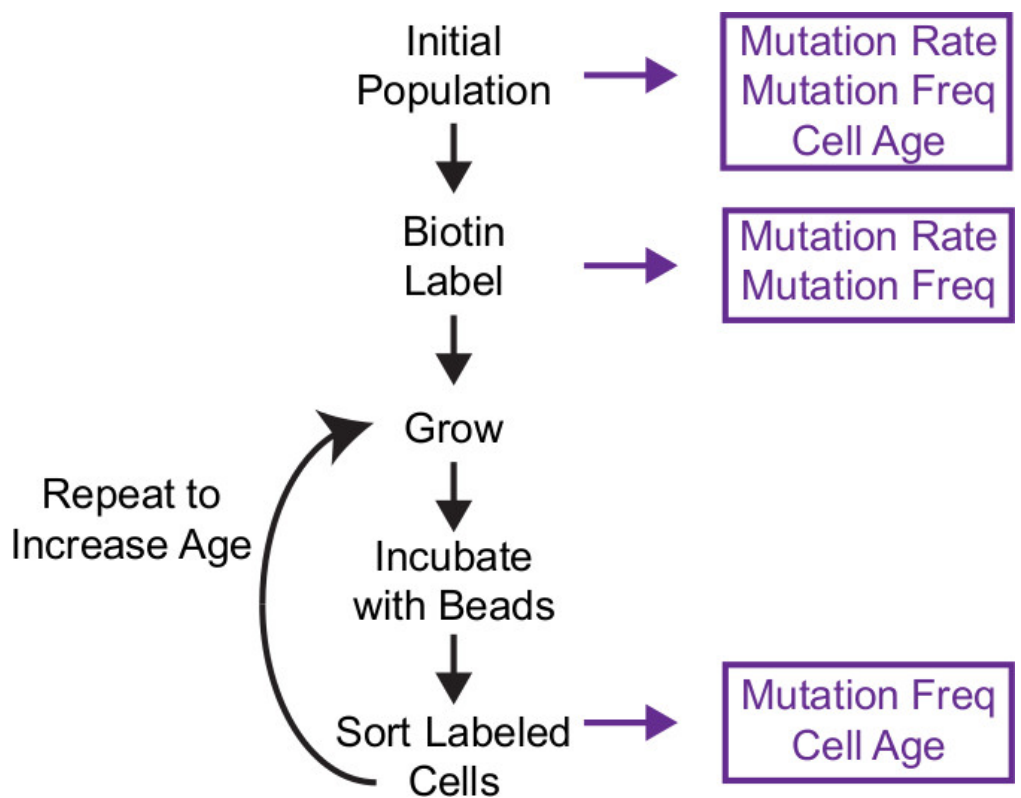

Figure 1. Flow diagram of the overall procedure for examining mutation accumulation during yeast replicative aging. Black text and arrows indicate major steps in the procedure. The curved arrow reflects that additional rounds of regrowth and sorting of the same cells are used to obtain progressively older cells. Purple arrows and text indicate steps at which the stated measurements are made. Freq - frequency. 
A

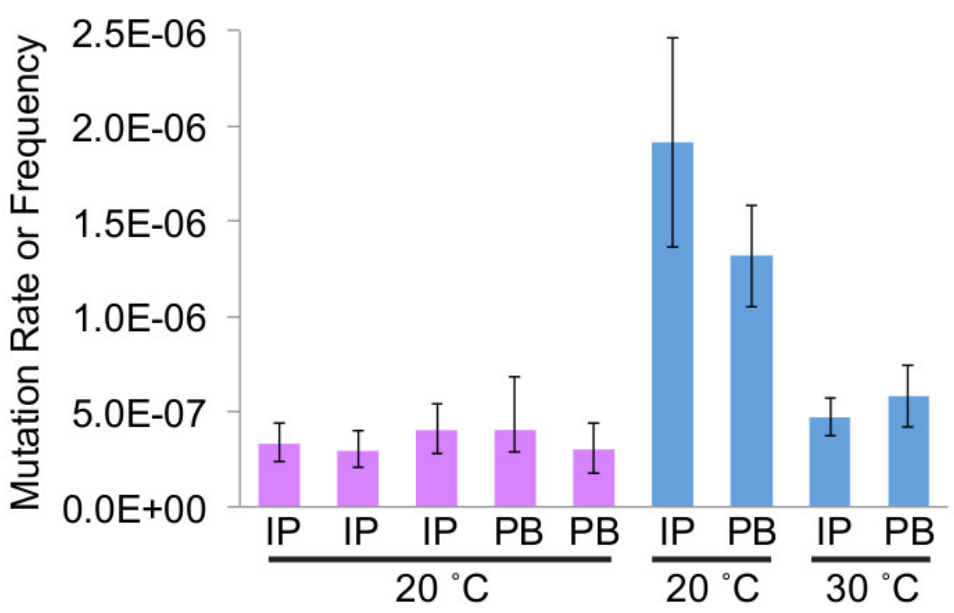

B

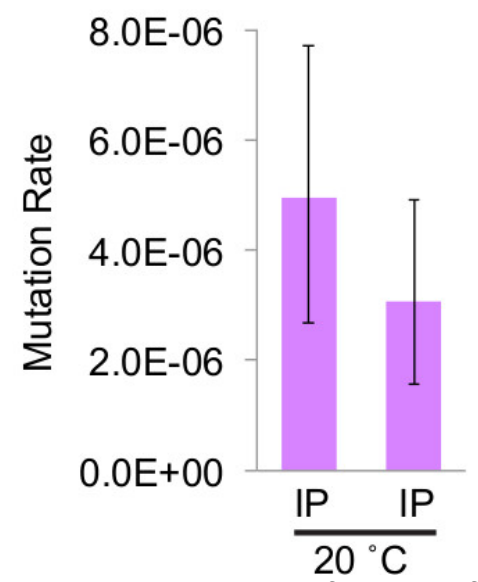

C

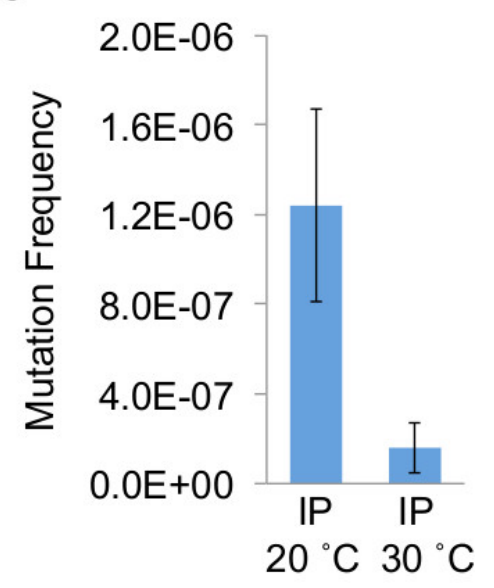

Figure 2. Determination of mutation frequency and rate in young cell populations. (A) Mutant colonies were selected by spreading cells on SC-arg + canavanine medium to select for loss-of-function mutations in the CAN1 gene and compared to the total number of viable cells spread on selective medium to calculate frequencies/rates. Cells from initial populations before biotin labeling (IP) or after biotin labeling (PB) were grown using YPD medium from an initial density of $5,000 \mathrm{cells} / \mathrm{ml}$ to near saturation. Lavender columns indicate rate measurements and blue columns represent frequency measurements made at indicated temperatures $\left(20^{\circ} \mathrm{C}\right.$ and $\left.30^{\circ} \mathrm{C}\right)$. Sets of seven replicate cultures were grown for each trial and two to five independent trials were performed. Individual rate values calculated for independent trials are shown, and error bars for these trials represent $95 \%$ confidence intervals determined using an online calculator ${ }^{22}$. Frequencies represent means and standard deviations. (B) CAN1 mutation rates obtained and represented as described for part A using a strain with CAN1 on the right arm of chromosome VIII. (C) Mutation frequencies obtained following selection for mutant colonies on 5-FOA using a strain with the URA3 gene located on the right arm of chromosome VIII. Methods were otherwise as for part A, and the means and standard deviations for three or four independent trials are shown. 


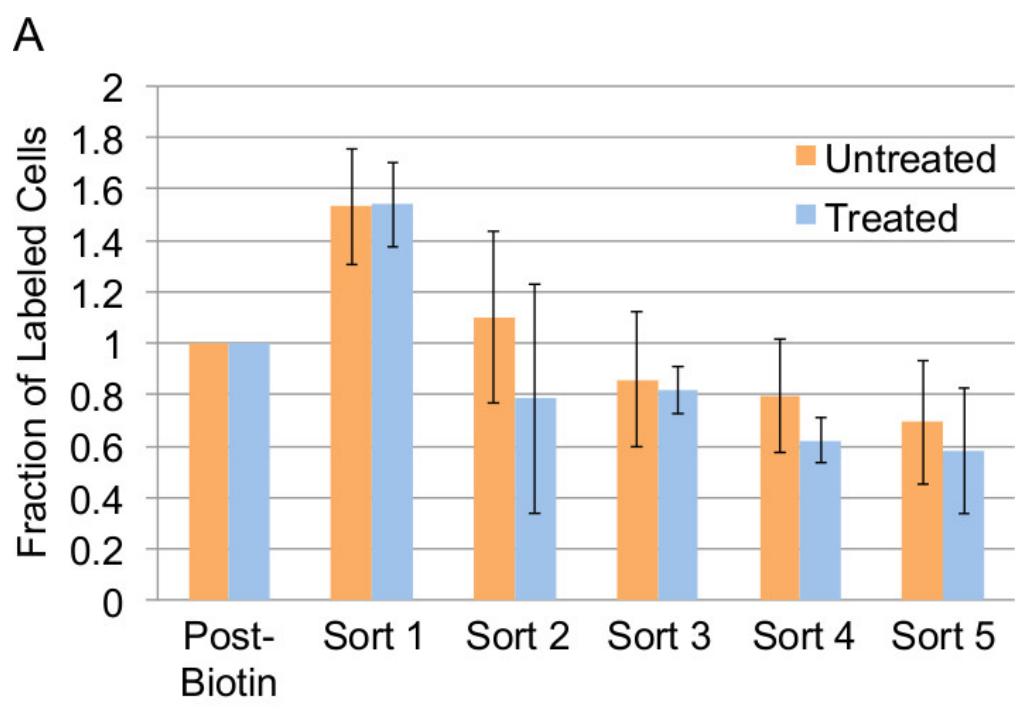

B

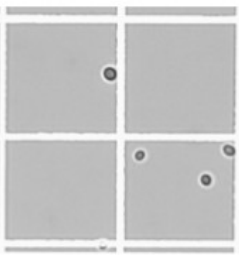

Post-Biotin

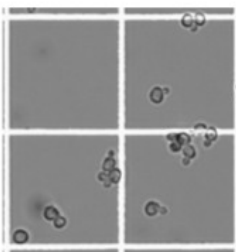

Sort 3

Mother Cells

Figure 3. Example sorting efficiency determined by calculating the number of eluted cells after each round of sorting. (A) The total number of cells in each starting population after biotin labeling was set to one, and the total number of cells present in the eluted samples from each round of magnetic cell sorting was divided by those initial values to obtain the fraction of labeled cells. Total cell numbers were determined from cell counts obtained using a hemocytometer. Orange columns represent the mean and standard deviation for three independent trials following the standard protocol. Blue columns represent the mean and standard deviation for two independent trials in which cells were treated with $1 \mathrm{mM}$ hydrogen peroxide for $30 \mathrm{~min}$ immediately after the first sort. (B) Size and morphology of cells after biotin labeling (post-biotin) and mother cells recovered after the third round of magnetic sorting (sort 3 mother cells) shown using standard bright field microscopy and a 20X objective. White lines in the backgrounds are the lines that bound the smallest squares visible on a standard hemocytometer. Please click here to view a larger version of this figure. 


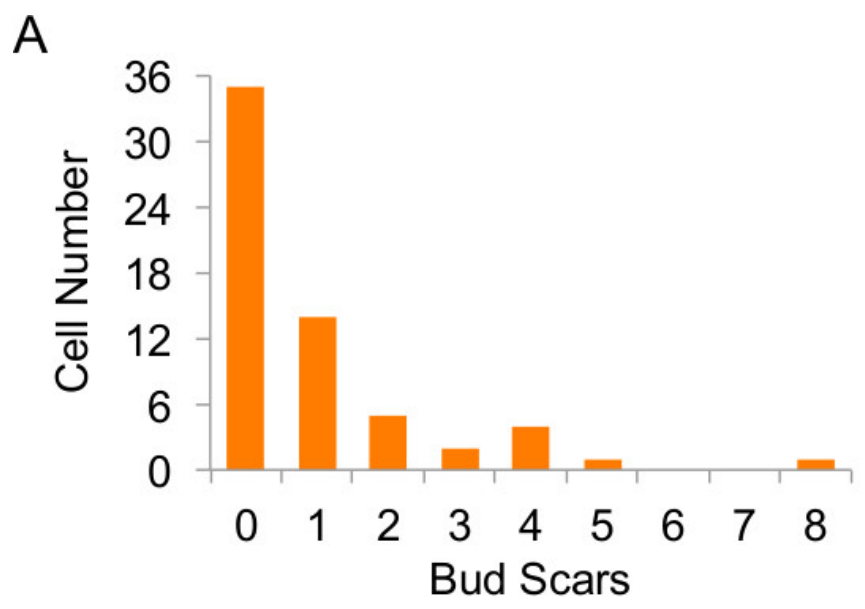

B

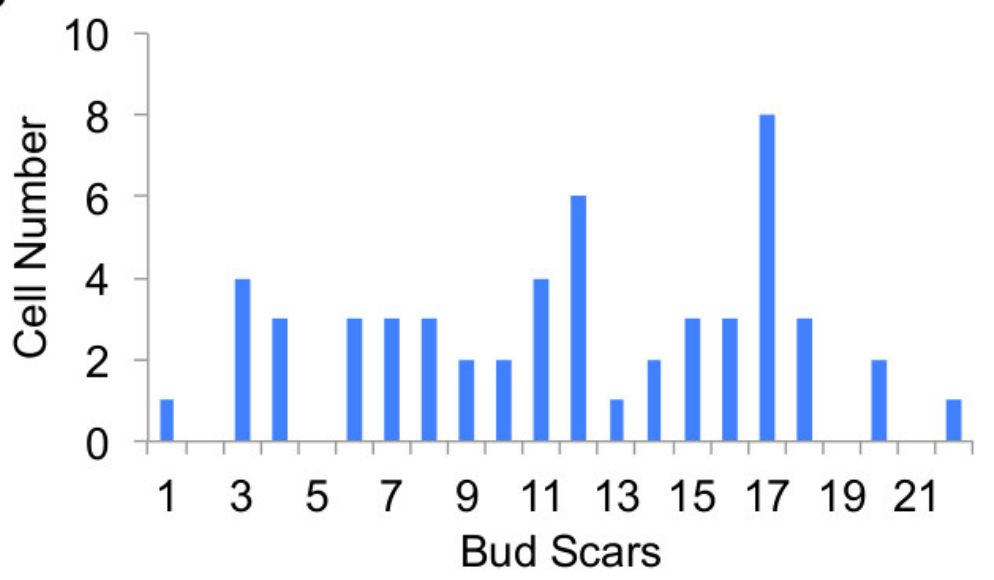

C

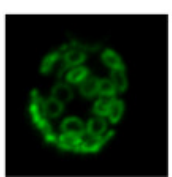

Figure 4. Determination of replicative age using manual bud scar counting. Confocal microscopy was used to count bud scars on individual cells labeled with a WGA-fluorescent conjugate (excitation at $488 \mathrm{~nm}$ and detection with $458 / 543 \mathrm{~nm}$ band pass and $505 \mathrm{~nm}$ long pass filter combination). (A) Manual bud scar counts of cells from the flow through (daughter cells) following the third round of magnetic sorting ( $n=62)$. (B) Manual bud scar counts of eluted cells (mother cells) following the third round of magnetic sorting $(n=54)$. (C) Mother cells retained following the third round of magnetic sorting photographed using a $63 \mathrm{X}$ oil immersion objective with $3 \mathrm{X}$ zoom after staining with a WGA-fluorescent conjugate. Please click here to view a larger version of this figure. 
A

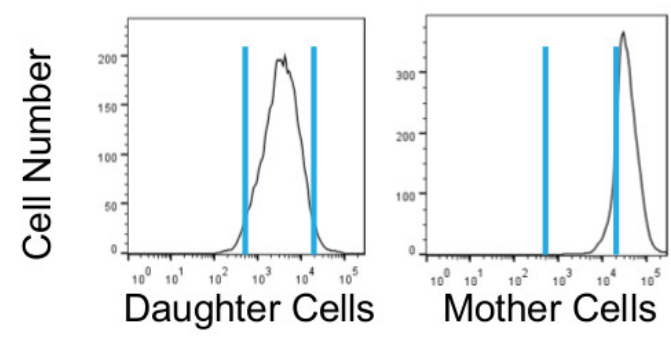

\section{Fluorescence Intensity}

B

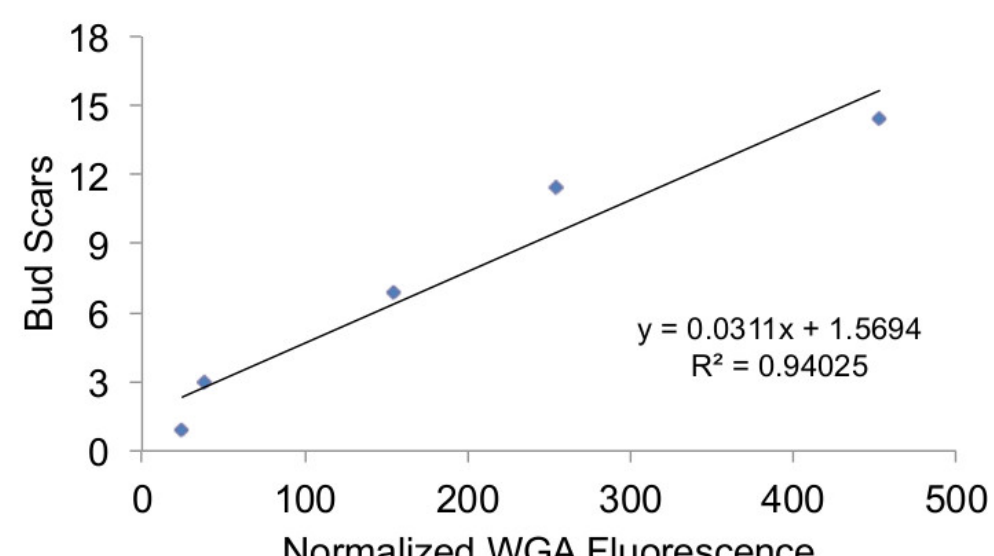

Figure 5. Determination of replicative age using flow cytometry. Samples of 10,000 cells stained with a WGA-fluorescent conjugate were analyzed by flow cytometry using excitation at $488 \mathrm{~nm}$ and detection with a $530 / 30$ band pass and 505 long pass filter set. (A) Histograms depicting the numbers of cells with specific WGA-fluorescence intensities for a daughter cell population or the eluate (mother cells) after three rounds of magnetic sorting. Populations correspond to those analyzed in Figure 4. Blue vertical lines indicate the corresponding position for the majority of the daughter cells on the mother cell histogram. (B) The normalized geometric mean for fluorescence signal of each population shown in A and three additional population of cells (average ages 3.0,6.9, and 14.4) was calculated as the ratio of the geometric mean of the stained cells and the geometric mean of the appropriate unstained cell population. These values are plotted in comparison to the average number of bud scars for each total population (Figure $\mathbf{4}$ and data not shown). The $\mathrm{R}^{2}$ value for the trend line of this comparison is given on the graph. 
A

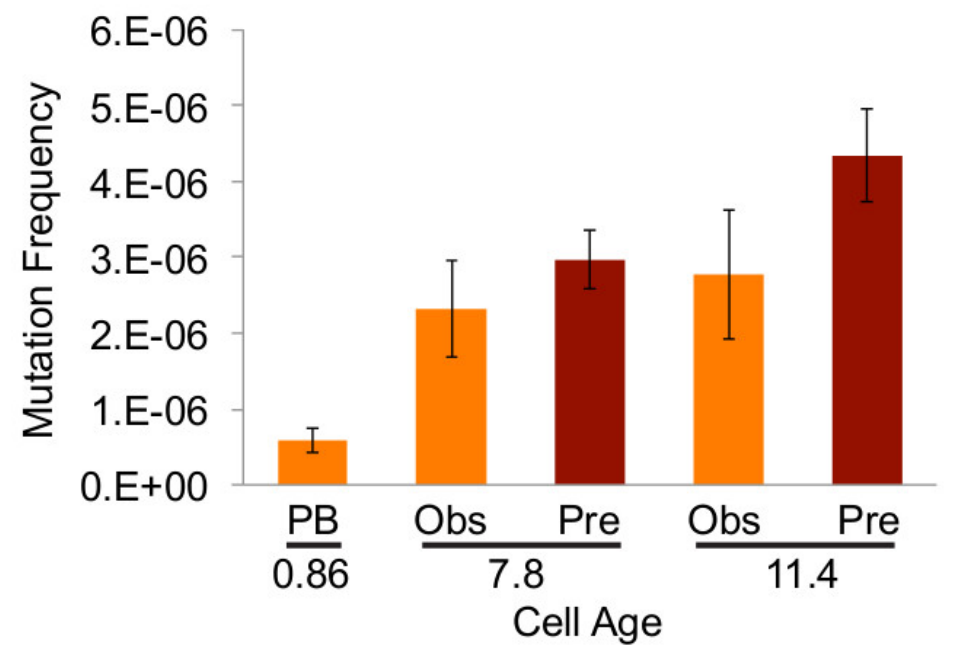

B

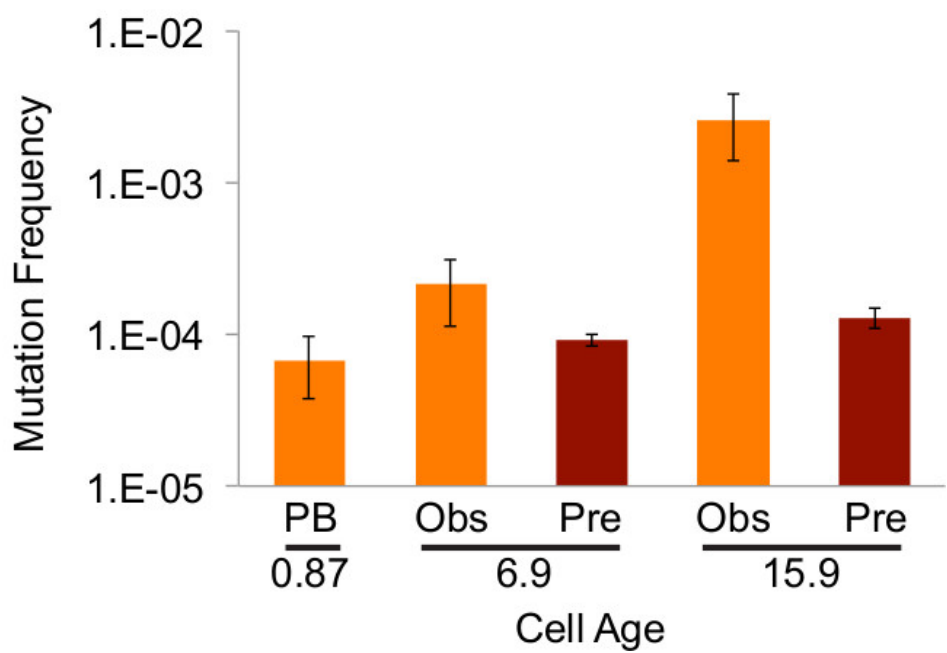

Figure 6. Comparison between predicted and observed mutation frequencies during replicative aging. Cells with mutations in the CAN1 gene were selected as described for Figure 2 using strains with CAN1 at its normal location on chromosome $\mathrm{V}$ (A) or on the right arm of chromosome VIII (B). Cells were grown on solid medium at $30^{\circ} \mathrm{C}$ prior to biotin labeling and at $20^{\circ} \mathrm{C}$ thereafter. Observed frequency values are shown with orange columns (Obs) and predicted frequencies for aged cells are shown with brown columns (Pre). The first column for each graph shows the mean and standard deviation of the initial mutation frequency after biotin labeling for four independent trials (PB). Numbers below the columns indicate the average number of bud scars for each population (Cell Age). Independent predicted values were determined as described in section 7 of the protocol using each of the initial rate values shown in Figure 2A (IP columns) and 2B. These independent values were then averaged. Observed values are means of three trials. Error bars indicate standard deviation.

\section{Discussion}

This protocol combines multiple methods to enable resources and approaches available in the Saccharomyces cerevisiae model system to be efficiently applied to the study of genome instability during mitotic cell aging. A wide variety of assays to quantify different forms of genome instability through phenotypic selection could be combined with magnetic sorting to study possible age-specific changes in accumulation of particular forms of mutations or chromosome rearrangements. While whole-genome sequencing approaches could provide more information about the overall types of changes occurring in a genome with age, the ability to use phenotypic selection systems to obtain mutation rate measurements and to preferentially isolate specific types of genetic changes are important advantages for studying mechanistic aspects of aging-related genome instability. Streamlining the determination of cell age and the potential to make parallel measurements of physiological changes through flow cytometry provide efficient means to correlate genome instability with other cellular changes during specific age ranges. Determination of potential age-dependent changes in rates of accumulating mutations requires: 1) careful determination of rates in young cell populations, 2) accurate determination of cell age, and 3) the ability to reliably enrich sufficiently large populations of mother cells to reproducibly measure genome instability in old age. This approach allows the yeast model system to contribute to defining underlying mechanisms responsible for age-dependent changes in rates and/or frequencies of genome instability in mitotically active cells. Furthermore, genetic and environmental factors can be rapidly assessed for contributions to age-dependent genome instability. Ultimately, these characterizations could lead to development of models that account for the manner in which mutations accumulate during replicative aging. 
This method depends on the ability to detect mutations or other types of genome instability through the appearance of selectable phenotypes to measure rates of such events. However, creativity in assay design can permit many aspects of genome instability to be investigated. For instance, the URA3 and CAN1 genes can be placed at different genomic sites to test any influences of genomic location on results. Reversion of specific non-functional gene alleles to functional gene alleles could test particular mutational processes. Also, introduction of multiple inactive alleles of a gene or flanking a gene with repeat sequences could be used to examine recombination events through restoration or loss of gene function, respectively. Fluctuation tests are the standard approach for determining rates of these various genome instability events ${ }^{9}$. The protocol is written using the well-accepted and relatively straightforward Lea-Coulson median estimator to determine mutation rate to make it more accessible to diverse researchers, even though the MSS-maximum likelihood estimator method is considered the best approach for determining mutation rates ${ }^{9}$. The MSS-maximum likelihood estimator has been previously reviewed in detail ${ }^{9}$, and can be used as an alternative method, but requires more sophisticated calculations. Alternatively, free software to calculate mutation rates with this method has been made available $^{22}$. Obtaining reproducible measures of mutation rates requires attention to a few aspects of experimental design, including using sufficient replicate cultures, inoculating cultures at low enough initial cell densities that population size increases by 10,000-fold or more, and choosing appropriate culture volumes so that the entire population of cells can be spread onto selective medium. For the latter point, a previously described formula can be used to adjust the mutation rate based on the fraction of culture tested ${ }^{9}$. Variation in growth rate of cultures can complicate the determination of a reproducible mutation rate, and a modified median-based estimator that may yield more reproducible results in such situations has been recently described ${ }^{23}$.

The ability to accurately measure cell age is another factor that is important for establishing whether genome instability frequencies in old cells are different from the values expected due to the rate of events in young cells. We have found WGA staining to be preferable to calcofluor white staining, both in terms of background and flexibility, since WGA is available conjugated to different fluorescent molecules. This flexibility can provide more opportunities for simultaneous measurements of other cell characteristics with fluorescent reagents. Initial work to establish a relationship between signal intensity for the WGA staining and the corresponding number of bud scars on cells can then lead to a more rapid determination of cell age through flow cytometry. This approach also allows use of larger population sizes than would be typically examined by microscopy for improved reproducibility of measurements. While all age determinations could be made through microscopic examination of the cells, we feel that the flow cytometry approach facilitates rapid screening of factors that influence age-dependent genome instability.

In addition to measuring cell age reliably, consideration needs to be given to the number of cell divisions mother cells are allowed to undergo with each successive round of growing and sorting cells. Use of a smaller initial population size or a larger culture volume could allow cells to undergo more cell divisions before reaching the desired cell density. For example, other researchers have used only two rounds of sorting to obtain very old yeast cells ${ }^{16}$, which has the obvious advantage of obtaining old cells with fewer experimental steps. When considering whether to increase culture volume to allow the cells to complete more cell divisions prior to sorting, keep in mind the limit on the total number of cells that can be processed in a single column. Use of a larger culture volume may necessitate the use of multiple columns to sort one cell population. Also, if cells undergo fewer rounds of cell division between sorts/collection points, then there are more opportunities to observe deviations from expected mutation frequencies at distinct time points during lifespan. For instance, sampling only at early and late time points during lifespan may produce data indicating a steady increase in mutation frequency, but sampling at additional intermediate times during lifespan may show that mutation frequency increases quickly and then plateaus. However, since this protocol isolates old mother cells, there is an opportunity to obtain a more direct measure of changes in mutation rates with age. Fluctuation tests could be performed using mother cells of different ages to determine whether the mutation rate will differ from the rate obtained using young cells. While the cultures for these fluctuation tests will become a mixture of old and young cells as they grow, any deviations from rates obtained starting with young cells could be attributed to the use of an older starting population.

The ability to reproducibly obtain a large enough population of aged mother cells to accurately measure genome instability is critical to the success of this approach. While the protocol describes the use of a particular magnetic sorting system for this purpose, other groups have sorted yeast mother cells with alternative systems ${ }^{14,18}$ (see Table of Materials). Such alternative systems should also work with this method, though some optimization of the manufacturers' protocols could be required. Regardless of the specific system used, the population size required differs depending on the frequency of the type of mutation or genome rearrangement chosen for study. At minimum, there must be enough mother cells to obtain at least one mutant colony per population to calculate a mutation frequency. In practice, results can be quite variable if only zero to five mutant colonies are expected from the population size, and even a small increase in population size, so that five to ten mutant colonies are expected, can greatly improve reproducibility. When biotin labeling a starting population, the recovery efficiency for each sort needs to be taken into account to help identify the appropriate population size necessary to measure mutation frequency in old mother cells. With $90 \%$ recovery of mother cells after each sort, only $59 \%$ of the original population would be recovered after five rounds of growth and sorting. This drops to $\sim 33 \%$ recovery of the original population after five rounds of growth and sorting if only $80 \%$ of labeled mother cells are recovered during each sort. Measuring and maximizing recovery is crucial when measuring low frequency events, since a two to three fold decrease in population size could easily lead to unreliable numbers of mutant colonies per population.

There are numerous options for expanding on or modifying this protocol to obtain a broader understanding of genome instability during mitotic cell aging. Simple examples include analyzing how alterations in gene functions, media changes, or other environmental condition changes alter the accumulation of mutations with age. Mutation rates for young cells with altered gene function or in the appropriate condition would be measured and used to determine whether mutations accumulate differently than predicted by the rate values and differently than in control cell populations. Cell populations at different points during replicative aging could be exposed to specific stressors, such as reactive oxygen species or DNA damaging agents. A transient and mild exposure to oxidative stress did not substantially alter the ability to perform cell sorting (Figure 3), but harsher stresses may complicate recovery of cells. Preliminary experiments would be necessary to verify that mother cells can still be recovered efficiently after specific stresses. Additionally, the cellular characteristics of isolated mother cells could be analyzed in detail, including levels of reactive oxygen species, mitochondrial and vacuolar morphology, and other physiological characteristics that are associated with aging ${ }^{3}$. Furthermore, the mother cells could be a starting population for a further treatment or manipulation. Since mother and daughter cells are physically separated during the procedure, the daughter cells are also still available for analysis. For example, changes in physiological characteristics of daughter cells or the asymmetric inheritance of damaged macromolecules between yeast mother and daughter cells ${ }^{24}$ could be compared to the timing of any interesting changes in mutation frequencies/rates. Mutation frequencies for daughter cell populations could also be obtained in order to try to model whether there is asymmetric inheritance of mutations during replicative aging. In summary, combining 
enrichment of mother cells through magnetic cell sorting with fluctuation tests enables the advantages of the S. cerevisiae model system to be efficiently applied to investigating mechanisms responsible for accumulation of genetic changes during mitotic cell aging.

\section{Disclosures}

The authors have nothing to disclose.

\section{Acknowledgements}

This work was supported in part by grant R00AG031911 from the National Institute on Aging of the National Institutes of Health to P. H. M. The content of this article does not necessarily reflect official views of the National Institutes of Health.

\section{References}

1. Maslov, A. Y., \& Vijg, J. Genome instability, cancer and aging. Biochim Biophys Acta. 1790, 963-969, doi:10.1016/j.bbagen.2009.03.020 (2009).

2. Maher, R. L., Branagan, A. M., \& Morrical, S. W. Coordination of DNA replication and recombination activities in the maintenance of genome stability. J Cell Biochem. 112, 2672-2682, doi:10.1002/jcb.23211 (2011).

3. Longo, V. D., Shadel, G. S., Kaeberlein, M., \& Kennedy, B. Replicative and chronological aging in Saccharomyces cerevisiae. Cell Metab. 16, 18-31, doi:10.1016/j.cmet.2012.06.002 (2012).

4. Madia, F., Gattazzo, C., Fabrizio, P., \& Longo, V. D. A simple model system for age-dependent DNA damage and cancer. Mech Ageing Dev. 128, 45-49, doi:10.1016/j.mad.2006.11.009 (2007).

5. Madia, F. et al. Oncogene homologue Sch9 promotes age-dependent mutations by a superoxide and Rev1/Polzeta-dependent mechanism. $J$ Cell Biol. 186, 509-523, doi:10.1083/jcb.200906011 (2009).

6. Lee, S. S., Avalos Vizcarra, I., Huberts, D. H., Lee, L. P., \& Heinemann, M. Whole lifespan microscopic observation of budding yeast aging through a microfluidic dissection platform. Proc Natl Acad Sci U S A. 109, 4916-4920, doi:10.1073/pnas.1113505109 (2012).

7. Xie, Z. et al. Molecular phenotyping of aging in single yeast cells using a novel microfluidic device. Aging Cell. 11, 599-606, doi:10.1111/ j.1474-9726.2012.00821.x (2012).

8. McMurray, M. A., \& Gottschling, D. E. An age-induced switch to a hyper-recombinational state. Science. 301, 1908-1911, doi:10.1126/ science.1087706 (2003).

9. Foster, P. L. Methods for determining spontaneous mutation rates. Methods Enzymol. 409, 195-213, doi:10.1016/S0076-6879(05)09012-9 (2006).

10. Boeke, J. D., LaCroute, F., \& Fink, G. R. A positive selection for mutants lacking orotidine-5"-phosphate decarboxylase activity in yeast: 5fluoro-orotic acid resistance. Mol Gen Genet. 197, 345-346 (1984).

11. Whelan, W. L., Gocke, E., \& Manney, T. R. The CAN1 locus of Saccharomyces cerevisiae: fine-structure analysis and forward mutation rates. Genetics. 91, 35-51 (1979).

12. Lindstrom, D. L., \& Gottschling, D. E. The mother enrichment program: a genetic system for facile replicative life span analysis in Saccharomyces cerevisiae. Genetics. 183, 413-422, 411SI-413SI, doi:10.1534/genetics.109.106229 (2009).

13. Lindstrom, D. L., Leverich, C. K., Henderson, K. A., \& Gottschling, D. E. Replicative age induces mitotic recombination in the ribosomal RNA gene cluster of Saccharomyces cerevisiae. PLoS Genet. 7, e1002015, doi:10.1371/journal.pgen.1002015 (2011).

14. Feser, J. et al. Elevated histone expression promotes life span extension. Mol Cell. 39, 724-735, doi:10.1016/j.molcel.2010.08.015 (2010).

15. Afonso, B., Silver, P. A., \& Ajo-Franklin, C. M. A synthetic circuit for selectively arresting daughter cells to create aging populations. Nucleic Acids Res. 38, 2727-2735, doi:10.1093/nar/gkq075 (2010).

16. Smeal, T., Claus, J., Kennedy, B., Cole, F., \& Guarente, L. Loss of transcriptional silencing causes sterility in old mother cells of $S$. cerevisiae. Cell. 84, 633-642 (1996).

17. Lam, Y. T., Aung-Htut, M. T., Lim, Y. L., Yang, H., \& Dawes, I. W. Changes in reactive oxygen species begin early during replicative aging of Saccharomyces cerevisiae cells. Free Radic Biol Med. 50, 963-970, doi:10.1016/j.freeradbiomed.2011.01.013 (2011).

18. Dang, W. et al. Histone H4 lysine 16 acetylation regulates cellular lifespan. Nature. 459, 802-807, doi:10.1038/nature08085 (2009).

19. Amberg, D. C., Burke, D. J., \& Strathern, J. N. Methods in Yeast Genetics: A Cold Spring Harbor Laboratory Course Manual, 2005 Edition. Cold Spring Harbor Laboratory Press, (2005).

20. Brachmann, C. B. et al. Designer deletion strains derived from Saccharomyces cerevisiae S288C: a useful set of strains and plasmids for PCR-mediated gene disruption and other applications. Yeast. 14, 115-132, doi:10.1002/(SICI)1097-0061(19980130)14:2<115::AIDYEA204>3.0.CO;2-2 (1998).

21. Maxwell, P. H., Burhans, W. C., \& Curcio, M. J. Retrotransposition is associated with genome instability during chronological aging. Proc Natl Acad Sci U S A. 108, 20376-20381, doi:10.1073/pnas.1100271108 (2011).

22. Hall, B. M., Ma, C. X., Liang, P., \& Singh, K. K. Fluctuation analysis CalculatOR: a web tool for the determination of mutation rate using LuriaDelbruck fluctuation analysis. Bioinformatics. 25, 1564-1565, doi:10.1093/bioinformatics/btp253 (2009).

23. $\mathrm{Wu}, \mathrm{X}$. et al. A robust estimator of mutation rates. Mutat Res. 661, 101-109, doi:10.1016/j.mrfmmm.2008.11.015 (2009).

24. Aguilaniu, H., Gustafsson, L., Rigoulet, M., \& Nyström, T. Asymmetric inheritance of oxidatively damaged proteins during cytokinesis. Science. 299, 1751-1753, doi:10.1126/science.1080418 (2003). 